\title{
Association between the sonographer's experience and diagnostic performance of IOTA simple rules
}

\author{
Chun-ping Ning ${ }^{1}$, Xiaoli $\mathrm{Ji}^{2}$, Hong-qiao Wang ${ }^{1}$, Xiao-ying $\mathrm{Du}^{1}$, Hai-tao $\mathrm{Niu}^{3^{*}}$ and Shi-bao Fang ${ }^{3^{*}}$
}

\begin{abstract}
Background: To validate the clinical value of simple rules in distinguishing malignant adnexal masses from benign ones and to explore the effect of simple rules for experienced and less-experienced sonographers.

Methods: Patients with persistent adnexal masses were enrolled between November 2013 and December 2015. All masses were proven through histological examinations. Five sets of diagnoses were made and compared with one another. Diagnosis 1 was made, according to the simple rules, by a trainee with little clinical diagnostic experience. Diagnoses 2 and 3 were made by experienced and less-experienced sonographers, respectively, according to their clinical experiences. With diagnosis 1 as a reference, the two sonographers were asked to provide a second diagnosis, which were diagnoses 4 and 5. The efficiency of the five sets of diagnoses was compared using ROC curves.

Results: In total, 75 malignant (37.7\%) and 124 benign lesions (62.3\%) were enrolled in this study. The mean diameter of the benign masses was obviously smaller than that of the malignant ones $(6.8 \pm 3.4 \mathrm{~cm}$ vs. $9.3 \pm$ $4.9 \mathrm{~cm}, p<0.01)$. The malignant ratio in postmenopausal women was much higher $(66.1 \%)$ than that in the premenopausal population (25.7\%) $(p<0.0001)$. Totally, 156 of the 199 cases $(79.4 \%)$ resulted in conclusive diagnoses. Sensitivity and specificity were $98.4 \%$ and $73.9 \%$, respectively, among the conclusive cases. The area under the ROC curve (Az) for the simple rule diagnosis was significantly lower than that for the experienced sonographer diagnosis ( 0.85 vs. $0.96, p<0.0001)$; compared with the less-experienced sonographer, this difference was not significant ( 0.85 vs. $0.86, p=0.9776)$. No significant difference was found in the comparison between the diagnoses made by the experienced sonographer before and after referencing the simple rule diagnosis ( $A z, 0.96 \mathrm{vs}$. 0.97, $p=0.2055$ ). Using diagnosis 1 as a reference, the diagnostic performance of the less-experienced sonographer increased (from 0.86 to 0.92 , $p=0.012$ ); however, it was still lower than that of the experienced sonographer ( $A z, 96 \%$ vs. 92\%, $p=0.0241$ ).
\end{abstract}

Conclusions: The simple rules was an appealing method for discriminating malignant masses from benign ones, particularly for a less-experienced sonographer.

Keywords: Ultrasound, Adnexal, Mass, Diagnosis

\section{Background}

Numerous diseases both benign and malignant can present as adnexal masses, such as ovarian cancers, hydrosalpinx, chocolate cysts, ectopic pregnancy, and adnexal abscess. Plenty of treatment options were proposed thanks to the surgical advances. A wise selection relies on the correct evaluation of the mass before the operation. However, the

\footnotetext{
* Correspondence: ningchunping1222@163.com; fsb-62@163.com

${ }^{3}$ Urology Department, Affiliated Hospital of Qingdao University, No. 16 of

Jiangsu Road, Qingdao, Shandong, China

Full list of author information is available at the end of the article
}

noninvasive preoperative assessment remains a major challenge for gynecologists.

Ultrasound, particularly transvaginal ultrasound, has been considered as the first-line examination in gynecology. An experienced sonographer was able to distinguish benign from malignant masses according to the subjective evaluation of ultrasound findings $[1,2]$. However, a great diversity of the examiner's experience was noticed which could influence the diagnostic performance significantly [3]. Most of the time, the expertise of differential diagnoses was a kind of instinct,

(C) The Author(s). 2018 Open Access This article is distributed under the terms of the Creative Commons Attribution 4.0 International License (http://creativecommons.org/licenses/by/4.0/), which permits unrestricted use, distribution, and 
and it was quite difficult to be transferred directly to an examiner with less experience from an experienced sonographer. Plenty of efforts were made during the last decades to improve the diagnostic ability of transvaginal sonography, such as proposing a scoring system establishing a logistic regression model, using the support vector machines, and so on [4-7]. However, none of the methods was convenient enough to be used universally.

"Simple rules" was a new method proposed by a group of researchers in the International Tumor Analysis Association (IOTA). The main aim of the proposal was to increase the diagnostic performance of ultrasound [8]. The rules contained ten ultrasound examination features, five of which were benign and five of which were malignant. Thus far, several papers have validated the clinical value of the rules [9-12]. However, the IOTA simple rules have not been tested in the Chinese population. In China, sonographers are responsible for both scanning and diagnosing, and the expertise of sonographers varies a lot. Therefore, the question is whether there are any differences when the simple rules were used by different sonographers and how the simple rules affect diagnoses made by sonographers with different experience levels.

This study had two aims: first, to validate the clinical value of simple rules in differentiating malignant adnexal masses from benign ones, and second, to explore the effect of simple rules on experienced and less-experienced sonographers.

\section{Methods}

The study was conducted between November 2013 and December 2015. The protocol for using the patients' ultrasonic images and pathological results to assess the efficacy of IOTA simple rules in distinguishing benign adnexal masses from malignant ones was approved by the Ethics Committee. All participants provided written consent to participate in the research.

\section{Patients}

Patients who were admitted to the gynecological department of the Affiliated Hospital of Qingdao University and who were scheduled for surgeries because of adnexal masses (detected by gynecologic examination with/without ultrasonography, previously) were included in this study. When a patient had more than one adnexal mass, the larger or more complex mass was included. When the masses were similar both in volume and texture, we selected the one that was more easily accessible through transvaginal ultrasound.

The following exclusion criteria were followed: (1) pregnant women; (2) patients who refused both transvaginal and transrectal ultrasound examinations; (3) patients whose surgery date exceeded 30 days from the date of the ultrasound scan; (4) patients who accepted adjuvant therapy, such as chemotherapy or radiotherapy, before the surgery; and (5) patients whose masses were surgically removed at other medical centers.

\section{Image storing}

The study was conducted using advanced ultrasound equipment (Voluson E8 ultrasound machine, GE Medical Systems). According to the protocol, all participants underwent transvaginal or transrectal (for virgins or patients who refused transvaginal ultrasound examination) ultrasound examinations with a transvaginal probe (frequency, 6 13 MHz). When the mass was too large to be viewed entirely by transvaginal ultrasound, transabdominal ultrasound was employed (frequency, 3 5 MHz). All examinations were performed by an experienced sonographer, who has been working in the gynecologic ultrasound department for 5 years. The sonographer was asked to fully scan the adnexal mass following the guidance proposed by IOTA [13]. Digital images and video clips of the masses were stored in a hard drive for further evaluations. At least eight images and three clips were stored for each patient. The size of the mass was measured in real-time.

\section{Diagnoses}

A total of five sets of diagnoses were made and analyzed in this study. One set was made using the simple rules, and the other four sets were based on subjective assessments of the sonographers.

\section{Diagnosis based on simple rules}

The simple rule diagnosis (diagnosis 1 ) was made by an ultrasound trainee who had studied in the ultrasonic department for approximately 1 year and accepted a 3 -month real-time ultrasound training period under the supervision of an expert examiner. Before the evaluation, the trainee had undergone a theoretical course, including the terms, definitions, and measurements of the sonographic features [13], according to the simple rules proposed by IOTA [8].

The simple rules included two groups of features $(\mathrm{M}$ features and B features) and three rules. Rule 1-a "malignant" diagnosis was made when a mass was found conformed to one or more " $\mathrm{M}$ " features without any " $\mathrm{B}$ " features. Rule 2-a mass was considered benign if it has one or more $B$ features and no $M$ feature. Rule 3-if both $M$ features and $B$ features were present, or if none of the features was present, the simple rules yield an "inconclusive" result. Here, the features were listed in Table 1.

\section{Diagnoses based on subjective assessments}

Two sonographers, one with 11 years of experience and the other with 3 years of experience, were invited to 
Table 1 Malignant and benign features of the "simple rules"

\begin{tabular}{lll}
\hline M features & B features \\
\hline 1 Irregular solid tumor & Unilocular cyst \\
2 Ascites & Acoustic shadows \\
3 At least four papillary structures & Smooth multilocular tumor \\
4 Irregular multilocular solid tumor & $\begin{array}{l}\text { The presence of solid } \\
\text { with the largest diameter of at least } \\
\text { components for which the } \\
\text { largest solid component is }\end{array}$ \\
& $<7$ mm in the largest diameter \\
5 Very high color content on color & $\begin{array}{l}\text { No detectable blood flow on } \\
\text { Doppler examination }\end{array}$ & Doppler examination \\
\hline
\end{tabular}

review the images and perform a diagnosis (diagnoses 2 and 3), respectively. They were asked to classify the masses into five groups, according to the ultrasonic features: benign, possibly benign, undetermined, possibly malignant, and malignant. The two reviewers were blinded to the clinical and pathological information when they were assessing the cases. The diagnoses were locked as soon as they were made and could not be changed afterwards. Two months later, the two sonographers were asked to review the stored images (order disturbed) again and perform a second diagnosis (diagnoses 4 and 5), respectively, after learning the simple rules by reading the original paper published by the IOTA group [8]. In addition, they had no knowledge of the pathological or clinical information of the patients during evaluation. Only at this time they were encouraged to use diagnosis 1 as a reference. Diagnosis 2 and diagnosis 4 were made by an experienced sonographer before and after referencing the simple rule diagnosis. Diagnosis 3 and diagnosis 5 were made by a less-experienced sonographer before and after referencing the simple rule diagnosis.

\section{Reference standard}

Pathological examinations were considered as the golden standard. An experienced pathologist was invited to examine the pathological specimens and to provide a final diagnosis, according to the criteria recommended by the International Federation of Gynecology and Obstetrics [14]. The pathologists had no knowledge of the ultrasonic diagnosis. The borderline masses and masses with low malignant potential were classified into the malignant group too. In benign cases which have no pathological specimens, intraoperative findings made by the surgeons were used as the final diagnoses.

\section{Statistical analysis}

For the subjective assessments, the "benign" and "possible benign" results were considered to be negative, while "malignant" and "possible malignant" were considered to be positive. Diagnostic performance was expressed as the sensitivity (Se), specificity (Sp), positive predictive value (PPV), negative predictive value (NPV), and accuracy (Ac). We compared the diagnostic efficiency of the simple rules with subjective assessments made by the experienced and less-experienced sonographers. We also assessed the performance when the simple rules were used as a second diagnosis by comparing the efficiency of the diagnoses made by the two sonographers, with and without using the simple rules as a reference.

Both SPSS (version 18.0, SPSS Inc., Chicago, IL) and MedCalc were used in the statistical analyses. Student's $t$ test was used to examine the differences between the numeric parameters, which were expressed as the mean \pm standard deviation. The Se, Sp, PPV, NPV, and Ac were calculated for each set of diagnoses. Receiver operating characteristic (ROC) curves were established, and the area under the ROC curves $(\mathrm{Az})$ was compared according to the method proposed by DeLong et al. [15]. McNemar's test was used to check for the statistically significant differences in a paired binomial proportion. A $p<0.01$ was considered as significantly different.

\section{Results}

During the study period, there were 373 eligible patients. A total of 174 were excluded for the following reasons: pregnancy $(n=11)$, patients refused both transvaginal and transrectal ultrasound examinations $(n=34)$, patients accepted surgeries 30 days later than the ultrasound scan $(n=46)$, patients accepted chemotherapy or radiotherapy before the surgery $(n=28)$, and masses were surgically removed in other medical centers $(n=55)$. Ultimately, 199 patients (mean age $45.1 \pm 13.7$, range 17 89 years) were included in this study, 59 of whom were postmenopausal.

Pathological examinations confirmed that there were 75 malignant (37.7\%) and 124 benign lesions (62.3\%), and the ratio of benign to malignant tumors was $1.65: 1$. The malignant percentage was much higher (66.1\%) in postmenopausal patients than that in the premenopausal population $(25.7 \%) \quad(p<0.0001)$. Detailed pathological types were listed in Table 2 . The mean diameter of the masses was $7.7 \pm 4.2 \mathrm{~cm}$ (range, $1.0 \sim 23.2 \mathrm{~cm}$ ); the mean diameter of the benign tumors was obviously smaller than that of the malignant ones $(6.8 \pm 3.4 \mathrm{~cm}$ vs. $9.3 \pm$ $4.9 \mathrm{~cm}, p<0.01$; power, 0.999).

The performances of the five sets of diagnoses are listed in Tables 3 and 4. The ROC curves for the five sets of diagnoses are shown in Fig. 1. The simple rules yielded a conclusive result for $79.4 \%(158 / 199)$ of the masses. Among the masses with conclusive results, the sensitivity was $98.4 \%$ and the specificity was $73.9 \%$. Compared with the subjective assessments performed by the experienced sonographer, the $\mathrm{Az}$ of diagnosis 1 was significantly lower ( 0.85 vs. $0.96, p<0.0001$, power $=0.981)$. However, the difference between the simple rule diagnosis and the diagnosis made by the less-experienced sonographer was not significant ( 0.85 vs. $0.86, p=0.9776$, power $=0.124$ ). 
Table 2 Detail pathological types of the enrolled masses

\begin{tabular}{|c|c|c|c|}
\hline Classifications & Pathological results & Number & Proportion (\%) \\
\hline \multirow[t]{12}{*}{ Benign } & Total & 124 & 62.3 \\
\hline & Teratoma & 43 & 21.6 \\
\hline & Chocolate cyst & 31 & 15.6 \\
\hline & Ovarian cystadenoma & 19 & 9.55 \\
\hline & Ectopic gestational mass & 8 & 4.02 \\
\hline & Para-ovarian cyst & 7 & 3.52 \\
\hline & Ovarian thecofibroma & 6 & 3.02 \\
\hline & Thecoma & 5 & 2.51 \\
\hline & Ovarian torsion & 1 & 0.50 \\
\hline & Accessory spleen & 1 & 0.50 \\
\hline & Abscess & 2 & 1.00 \\
\hline & $\begin{array}{l}\text { Isolated torsion of the } \\
\text { fallopian tube }\end{array}$ & 1 & 0.50 \\
\hline \multirow[t]{10}{*}{ Malignant } & Total & 75 & 37.7 \\
\hline & Cystadenocarcinoma & 42 & 21.1 \\
\hline & Ovarian borderline tumor & 15 & 7.54 \\
\hline & Endometrial cancer & 3 & 1.51 \\
\hline & Metastatic carcinoma & 2 & 1.00 \\
\hline & Yolk sac tumor & 2 & 1.00 \\
\hline & Granulosa cell carcinoma & 1 & 0.50 \\
\hline & Dysgerminoma & 1 & 0.50 \\
\hline & Immature teratoma & 7 & 3.52 \\
\hline & Carcinosarcoma & 2 & 1.00 \\
\hline
\end{tabular}

For the experienced sonographer, the conclusive ratio was $81.4 \%(162 / 199)$. Among the concluded cases, the primary diagnosis missed two malignant masses and yielded four false-positive diagnoses. With the help of the simple rules, 21 more cases were classified as conclusive. However, the diagnostic performance was similar (0.96 vs. 0.97 , $p=0.2055$, power $=0.241$ ) before and after using the simple rules as a reference. Comparison of the sensitivity and specificity of the two diagnoses (diagnoses 2 and 4) yielded no significant difference (sensitivity, $96.2 \%$ vs. $100 \%$; specificity, $96.3 \%$ vs. $94.0 \%, p>0.05$ ). Az of the diagnosis made by the experienced sonographer was obviously higher than that of the less-experienced sonographer (Az, 0.96 vs. $0.86, p<0.0001$, power $=0.966$ ).
For the less-experienced sonographer, 137 of the 199 cases were correctly diagnosed, with moderate sensitivity and specificity (72.4\% and $88.8 \%$ ). Using the simple rules as a reference, the diagnostic performance of the less-experienced sonographer increased (from 0.86 to $0.92, p=0.012$, power $=0.659)$; however, it remained lower than that of the experienced sonographer (Az, 96\% vs. $92 \%, p=0.0241$, power $=0.728$ ). The conclusive ratio showed no significant change $(82.9 \%$ vs. $81.4 \%, p=0.795)$ before and after using the simple rule diagnosis as a reference. The sensitivity of diagnosis 5 (made by the less-experienced sonographer with the help of the simple rules) was obviously higher than that of diagnosis $3(96.7 \%$ vs. $72.4 \%, p=0.012$ ).

\section{Discussion}

Adnexal masses are frequently found in both symptomatic and asymptomatic women at most ages. Benign and malignant masses may be found in adnexal structures. Once a mass has been detected, the physician is faced with the dilemma of how to manage the patient. Appropriate management should be based on the correct preoperative diagnosis. Ultrasound, particularly transvaginal ultrasound, remains the leading established tool to predict the nature of the adnexal masses [16].

In this study, we enrolled 199 adnexal masses detected over 3 years, including 11 types of benign masses and 9 types of malignant masses. We found that the malignant percentage in postmenopausal patients was higher than that in the premenopausal population, and the malignant masses were significantly larger than the benign masses. Thus, it is reasonable to pay more attention to older patients with large adnexal masses.

The simple rules were established by comparing the diagnostic performance with two logic regression models [8]. Up to now, plenty of studies $[17,18]$ have proved that the simple rules were suitable for about $76-89.3 \%$ adnexal tumors. In our study, the conclusive ratio of the simple diagnosis was $79.4 \%$. The ratio increased to $92.0 \%$ and $81.4 \%$ in experienced and less-experienced examiners, respectively. Therefore, we believe that the simple rules are a user-friendly tool for both experienced and less-experienced examiners. For the masses with conclusive results, the sensitivity in this study was lower

Table 3 Detail results of the five sets diagnoses

\begin{tabular}{|c|c|c|c|c|c|c|c|c|c|c|}
\hline \multirow{2}{*}{$\begin{array}{l}\text { Pathological } \\
\text { diagnosis }\end{array}$} & \multicolumn{2}{|c|}{ Diagnosis 1} & \multicolumn{2}{|c|}{ Diagnosis 2} & \multicolumn{2}{|c|}{ Diagnosis 3} & \multicolumn{2}{|c|}{ Diagnosis 4} & \multicolumn{2}{|c|}{ Diagnosis 5} \\
\hline & - & + & - & + & - & + & - & + & - & + \\
\hline- & 71 & 25 & 105 & 4 & 95 & 12 & 109 & 7 & 89 & 13 \\
\hline+ & 1 & 61 & 2 & 51 & 16 & 42 & 0 & 67 & 2 & 58 \\
\hline Inconclusive & 28 & 13 & 15 & 22 & 17 & 17 & 8 & 8 & 22 & 15 \\
\hline
\end{tabular}

Diagnosis 1 was made by a trainee according to the simple rules. Diagnoses 2 and 3 were made by an experienced and a less-experienced sonographer, respectively, according to their clinical experiences. Diagnoses 4 and 5 were made by the experienced and less-experienced sonographer, respectively, according to their experiences, with diagnosis 1 as a reference. "-" means "benign," "+" means "malignant" 
Table 4 Diagnostic performance of the five diagnoses

\begin{tabular}{|c|c|c|c|c|c|c|c|c|c|c|}
\hline Diagnoses & Conclusive ratio (\%) & Sensitivity (\%) & Specificity (\%) & PPV (\%) & NPV (\%) & $+\mathrm{LR}$ & $-L R$ & Correct ratio (\%) & $\mathrm{Az}$ & $95 \% \mathrm{Cl}$ \\
\hline Diagnosis 1 & 79.4 & 98.4 & 73.9 & 70.9 & 98.6 & 3.77 & 0.02 & $83.5 \%$ & 0.85 & $0.797 \sim 0.900$ \\
\hline Diagnosis 2 & 81.4 & 96.2 & 96.3 & 92.7 & 98.1 & 26 & 0.04 & 96.3 & $0.96^{*}$ & $0.923 \sim 0.983$ \\
\hline Diagnosis 3 & 82.9 & 72.4 & 88.8 & 77.8 & 85.6 & 6.46 & 0.31 & 83.0 & $0.86^{\#}$ & $0.798 \sim 0.90$ \\
\hline Diagnosis 4 & 92.0 & 100 & 94.0 & 90.5 & 100 & 16.7 & 0 & 96.2 & $0.97^{*^{\Delta}}$ & $0.934 \sim 0.988$ \\
\hline Diagnosis 5 & 81.4 & 96.7 & 87.3 & 81.7 & 97.8 & 7.61 & 0.04 & 90.7 & $0.92^{* \# \Delta} \boldsymbol{\Lambda}$ & $0.870 \sim 0.95$ \\
\hline
\end{tabular}

Diagnosis 1 was made by a trainee of ultrasound according to the simple rules. Diagnoses 2 and 3 were made by an experienced and a less-experienced sonographer, respectively, according to their experiences. Diagnoses 4 and 5 were made by the experienced and less-experienced sonographer, respectively, according to their experiences, with diagnosis 1 as a reference

$A z$ area under the ROC curve, $C l$ confidence interval, $P P V$ positive predictive ratio, NPV negative predictive ratio, $+L R$ positive likelihood ratio, $-L R$ negative likelihood ratio

*Compared with diagnosis $1, p<0.01$

"Compared with diagnosis $2, p<0.01$

${ }^{\triangle}$ Compared with diagnosis $3, p<0.01$

${ }^{\Delta}$ Compared with diagnosis $4, p<0.01$

than that in Nunes [19] reported in their meta-analysis, while the specificity was higher. This difference may be partly explained by the fact that this study was performed in a tertiary care university-affiliated hospital, which was considered to be the best hospital in Qingdao, and partly because the simple rule diagnosis was conducted by a trainee with little clinical experience.

The diagnostic performance of the experienced sonographer improved while the sensitivity and specificity remained unchanged when the simple rule diagnosis was used as a reference. The experienced sonographer missed two malignant masses and provided four false-positive diagnoses during the first round of diagnosis. With the help of the simple rules, there were seven false-positive diagnoses and no malignant masses were missed. The three new misdiagnosed cases eventually proved to be inflammatory masses. They were irregular solid masses with abundant blood supplies, and one of the patients had a small amount of ascite, which were diagnosed as malignant according to the simple rules. For the less-experienced sonographer, the sensitivity and specificity, as well as the Az, improved significantly with the help of the simple rule diagnosis. Consequently,

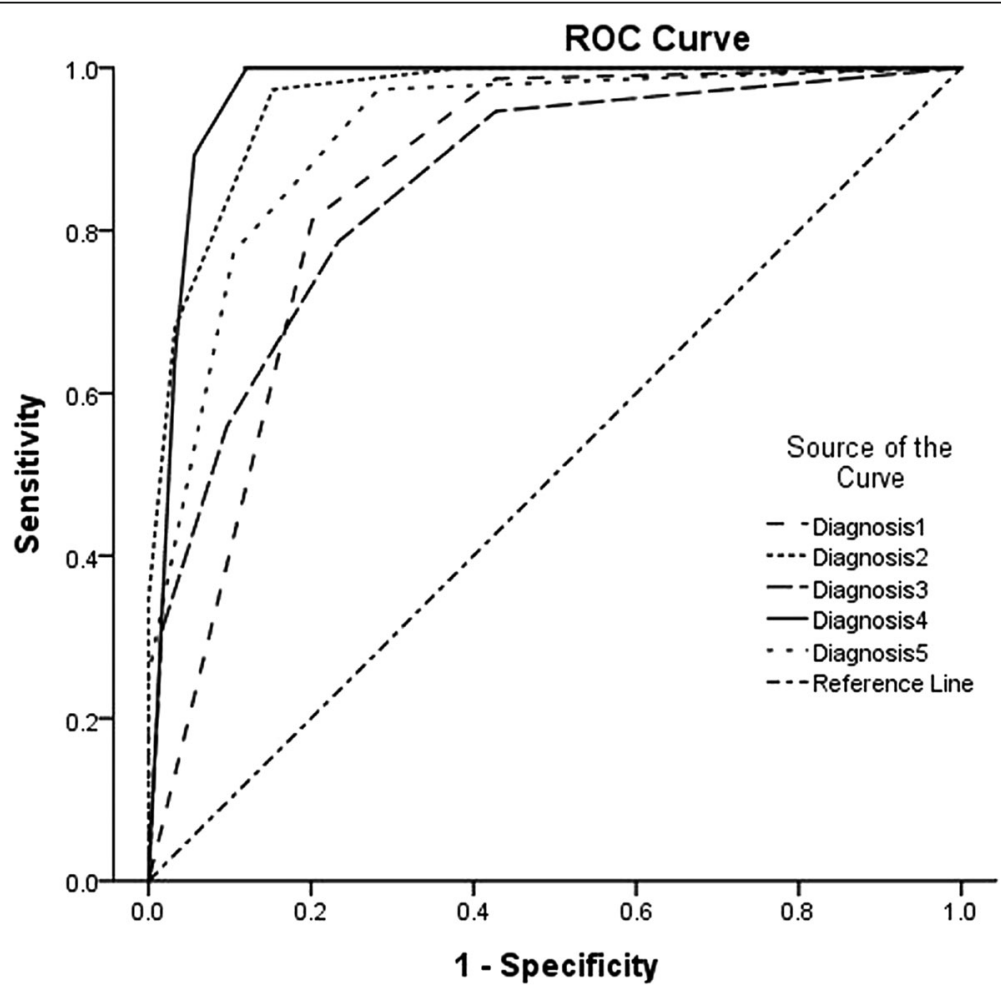

Fig. 1 The ROC curves for the five sets of diagnoses 
we believe that the simple rules may be more helpful for less-experienced examiners. However, the $\mathrm{Az}$ of diagnosis 4 (made by the less-experienced sonographer with the help of the simple rules) was still significantly lower than that of the experienced sonographer, implying that the clinical experience is crucial for the efficient diagnosis of adnexal masses.

This study has two disadvantages. First, the clinical information and laboratory results were not provided when the masses were assessed. Some of the masses, for example, ectopic pregnancy, could be correctly diagnosed if the results of HCG were provided. In clinical practices, such information could be obtained from inquisitions at the time of scanning. Second, only two sonographers were invited to participate in this study, one with 11 years of experience and the other with 3 years of experience. Examiners with various experience levels should be evaluated in further research.

\section{Conclusions}

In conclusion, this study was performed to demonstrate the differences in how the simple rules affect the diagnoses made by the sonographers with different experience levels. We found that the simple rules was more useful for the less-experienced sonographers. When the diagnosis is still inconclusive, it is wise to seek help from the experienced sonographers.

\section{Acknowledgements \\ We want to express our gratitude to the American Journal Experts for the professional editing of the manuscript.}

\section{Funding}

This study was partially funded by the National Natural Science Foundation of China (No. 81501477). This funding helped the design and collection of the manuscript. It will also support the publication of the article. This study was also partially funded by the post-doctoral application project of Qingdao.

\section{Availability of data and materials}

Please contact the author for data requests.

\section{Authors' contributions}

CPN contributed to the protocol/project development, data analysis, and manuscript writing/editing. $\mathrm{XLJ}$ contributed to the data analysis and statistics and revised the manuscript. HQW contributed to the data management and analysis. XYD contributed to the data collection and manuscript writing. HTN contributed to the supervision, statistical analysis, and manuscript editing. SBF contributed to the protocol/project development and supervision. All authors read and approved the final manuscript.

\section{Ethics approval and consent to participate}

The protocol for the use of patients' ultrasonic images and pathological results to evaluate the efficacy of IOTA simple rules to differentiate benign from malignant adnexal masses was approved by the Ethics Committee of the Medical College, Qingdao University (No. QDM-13-0517-U001). All the participants provided their written consents to participate in the research.

\section{Consent for publication}

Not applicable.

\section{Competing interests}

The authors declare that they have no competing interests.

\section{Publisher's Note}

Springer Nature remains neutral with regard to jurisdictional claims in published maps and institutional affiliations.

\section{Author details}

${ }^{1}$ Ultrasound Department, Affiliated Hospital of Qingdao University, Qingdao, Shandong, China. ${ }^{2}$ Ultrasound Department, Qingdao Women and Children Hospital, Qingdao, Shandong, China. ${ }^{3}$ Urology Department, Affiliated Hospital of Qingdao University, No. 16 of Jiangsu Road, Qingdao, Shandong, China.

Received: 22 December 2017 Accepted: 21 August 2018

Published online: 05 September 2018

\section{References}

1. Van Calster B, Timmerman D, Bourne T, Testa AC, Van Holsbeke C, Domali E, Jurkovic D, Neven P, Van Huffel S, Valentin L. Discrimination between benign and malignant adnexal masses by specialist ultrasound examination versus serum CA-125. J Natl Cancer Inst. 2007;99:1706-14.

2. Valentin L, Jurkovic D, Van Calster B, Testa A, Van Holsbeke C, Bourne T, Vergote I, Van Huffel S, Timmerman D. Adding a single CA 125 measurement to ultrasound imaging performed by an experienced examiner does not improve preoperative discrimination between benign and malignant adnexal masses. Ultrasound Obstet Gynecol. 2009;34:345-54.

3. Van Holsbeke C, Daemen A, Yazbek J, Holland TK, Bourne T, Mesens T, Lannoo L, Boes AS, Joos A, Van De Vijver A, et al. Ultrasound experience substantially impacts on diagnostic performance and confidence when adnexal masses are classified using pattern recognition. Gynecol Obstet Investig. 2010;69:160-8.

4. Sladkevicius $P$, Valentin L. Interobserver agreement in describing the ultrasound appearance of adnexal masses and in calculating the risk of malignancy using logistic regression models. Clin Cancer Res. 2015;21: 594-601.

5. Tailor A, Jurkovic D, Bourne TH, Collins WP, Campbell S. Sonographic prediction of malignancy in adnexal masses using an artificial neural network. Br J Obstet Gynaecol. 1999;106:21-30.

6. Ferrazzi E, Zanetta G, Dordoni D, Berlanda N, Mezzopane R, Lissoni AA. Transvaginal ultrasonographic characterization of ovarian masses: comparison of five scoring systems in a multicenter study. Ultrasound Obstet Gynecol. 1997;10:192-7.

7. Kaijser J, Sayasneh A, Van Hoorde K, Ghaem-Maghami S, Bourne T, Timmerman D, Van Calster B. Presurgical diagnosis of adnexal tumours using mathematical models and scoring systems: a systematic review and meta-analysis. Hum Reprod Update. 2014;20:449-62.

8. Timmerman D, Testa AC, Bourne T, Ameye L, Jurkovic D, Van Holsbeke C, Paladini D, Van Calster B, Vergote I, Van Huffel S, Valentin L. Simple ultrasound-based rules for the diagnosis of ovarian cancer. Ultrasound Obstet Gynecol. 2008;31:681-90.

9. Di Legge A, Testa AC, Ameye L, Van Calster B, Lissoni AA, Leone FP, Savelli L, Franchi D, Czekierdowski A, Trio D, et al. Lesion size affects diagnostic performance of IOTA logistic regression models, IOTA simple rules and risk of malignancy index in discriminating between benign and malignant adnexal masses. Ultrasound Obstet Gynecol. 2012;40:345-54.

10. Alcazar JL, Pascual MA, Olartecoechea B, Graupera B, Auba M, Ajossa S, Hereter L, Julve R, Gaston B, Peddes C, et al. IOTA simple rules for discriminating between benign and malignant adnexal masses: prospective external validation. Ultrasound Obstet Gynecol. 2013;42:467-71.

11. Tinnangwattana $D$, Vichak-Ururote L, Tontivuthikul P, Charoenratana $C$, Lerthiranwong T, Tongsong T. IOTA simple rules in differentiating between benign and malignant adnexal masses by non-expert examiners. Asian Pac J Cancer Prev. 2015;16:3835-8.

12. Alcazar JL, Pascual MA, Graupera B, Auba M, Errasti T, Olartecoechea B, RuizZambrana A, Hereter L, Ajossa S, Guerriero S. External validation of IOTA simple descriptors and simple rules for classifying adnexal masses. Ultrasound Obstet Gynecol. 2016;48:397-402.

13. Timmerman D, Valentin L, Bourne TH, Collins WP, Verrelst H, Vergote I, International Ovarian Tumor Analysis G. Terms, definitions and measurements to describe the sonographic features of adnexal tumors: a consensus opinion from the International Ovarian Tumor Analysis (IOTA) Group. Ultrasound Obstet Gynecol. 2000;16:500-5.

14. Heintz AP, Odicino F, Maisonneuve P, Quinn MA, Benedet JL, Creasman WT, Ngan HY, Pecorelli S, Beller U. Carcinoma of the ovary. FIGO 26th annual 
report on the results of treatment in gynecological cancer. Int J Gynaecol Obstet. 2006;95(Suppl 1):S161-92.

15. DeLong ER, DeLong DM, Clarke-Pearson DL. Comparing the areas under two or more correlated receiver operating characteristic curves: a nonparametric approach. Biometrics. 1988;44:837-45.

16. Meys EM, Rutten IJ, Kruitwagen RF, Slangen BF, Bergmans MG, Mertens HJ, Nolting E, Boskamp D, Beets-Tan RG, van Gorp T. Investigating the performance and cost-effectiveness of the simple ultrasound-based rules compared to the risk of malignancy index in the diagnosis of ovarian cancer (SUBSONiC-study): protocol of a prospective multicenter cohort study in the Netherlands. BMC Cancer. 2015;15:482.

17. Fathallah $\mathrm{K}$, Huchon C, Bats AS, Metzger U, Lefrere-Belda MA, Bensaid C, Lecuru F. External validation of simple ultrasound rules of Timmerman on 122 ovarian tumors. Gynecol Obstet Fertil. 2011;39:477-81.

18. Hartman CA, Juliato CR, Sarian LO, Toledo MC, Jales RM, Morais SS, Pitta DD, Marussi EF, Derchain S. Ultrasound criteria and CA 125 as predictive variables of ovarian cancer in women with adnexal tumors. Ultrasound Obstet Gynecol. 2012;40:360-6.

19. Nunes N, Ambler G, Foo X, Naftalin J, Widschwendter M, Jurkovic D. Use of IOTA simple rules for diagnosis of ovarian cancer: meta-analysis. Ultrasound Obstet Gynecol. 2014;44:503-14.

Ready to submit your research? Choose BMC and benefit from:

- fast, convenient online submission

- thorough peer review by experienced researchers in your field

- rapid publication on acceptance

- support for research data, including large and complex data types

- gold Open Access which fosters wider collaboration and increased citations

- maximum visibility for your research: over $100 \mathrm{M}$ website views per year

At $\mathrm{BMC}$, research is always in progress.

Learn more biomedcentral.com/submissions 